\title{
Will Spin-Relaxation Times in Molecular Magnets Permit Quantum Information Processing?
}

\author{
Arzhang Ardavan, Olivier Rival, John J.L. Morton, and Stephen J. Blundell \\ Clarendon Laboratory, Department of Physics, University of Oxford, OX1 3PU, United Kingdom \\ Alexei M. Tyryshkin \\ Department of Electrical Engineering, Princeton University, Princeton, New Jersey 08544, United States, USA \\ Grigore A. Timco and Richard E. P. Winpenny \\ Department of Chemistry, University of Manchester, Oxford Road, Manchester, M13 9PL, United Kingdom
}

(Received 4 October 2006; published 29 January 2007)

\begin{abstract}
Using $X$-band pulsed electron-spin resonance, we report the intrinsic spin-lattice $\left(T_{1}\right)$ and phasecoherence $\left(T_{2}\right)$ relaxation times in molecular nanomagnets for the first time. $\operatorname{In} \mathrm{Cr}_{7} M$ heterometallic wheels, with $M=\mathrm{Ni}$ and $\mathrm{Mn}$, phase-coherence relaxation is dominated by the coupling of the electron spin to protons within the molecule. In deuterated samples $T_{2}$ reaches $3 \mu$ s at low temperatures, which is several orders of magnitude longer than the duration of spin manipulations, satisfying a prerequisite for the deployment of molecular nanomagnets in quantum information applications.
\end{abstract}

DOI: 10.1103/PhysRevLett.98.057201

Certain computational tasks can be efficiently implemented using quantum logic, in which the informationcarrying elements are permitted to exist in quantum superpositions [1]. To achieve this in practice, a physical system that is suitable for embodying quantum bits (qubits) must be identified. Some proposed scenarios employ electron spins in the solid state, for example, phosphorous donors in silicon [2], quantum dots [3], heterostructures [4], and endohedral fullerenes $[5,6]$, motivated by the long electron-spin-relaxation times exhibited by these systems. An alternative electron-spin based proposal exploits the large number of quantum states and the nondegenerate transitions available in high spin molecular magnets $[7,8]$. Although these advantages have stimulated vigorous research in molecular magnets [9-11], the key question of whether the intrinsic spin-relaxation times are long enough has hitherto remained unaddressed. Here we show, using pulsed electron-spin resonance experiments on heterometallic wheels, that the relaxation times in molecular magnets can significantly exceed the duration of coherent manipulations, a prerequisite for the deployment of these systems in quantum information applications.

Molecular magnets comprising clusters of exchanged coupled transition metal ions have been studied extensively in recent years [12]. They can exhibit a substantial ground state spin with a large and negative zero-field splitting (ZFS), leading to a spontaneous magnetic moment parallel to the easy axis. In the absence of a magnetic field, the configurations in which the moment is "up" or "down" relative to the easy axis are degenerate, and this bistable nature has stimulated interest in the application of magnetic clusters as classical [13] or quantum [7-9,11] information elements.

Molecules in this class have been synthesized with widely varying properties, from the $S=10$ highly axial
PACS numbers: 75.50.Xx, 03.67.- a, 75.50.Tt, 76.30.-v

$\mathrm{Mn}_{12}$ acetate [14], to the diamagnetic ring $\mathrm{Cr}_{8} \mathrm{~F}_{8} \mathrm{Piv}_{16}$ $[15,16]$. A key recent chemical advance is the development of procedures for magnetically "doping" a diamagnetic cluster to synthesize paramagnetic molecules in a systematic and controllable way [17]. Thus, substituting a $\mathrm{Cr}^{3+}$ $(s=3 / 2)$ by a $\mathrm{Mn}^{2+}(s=5 / 2)$ or a $\mathrm{Ni}^{2+}(s=1)$ generates the $S=1 \mathrm{Cr}_{7} \mathrm{Mn}$ or the $S=1 / 2 \mathrm{Cr}_{7} \mathrm{Ni}$, respectively.

Many clusters have been investigated using thermodynamic probes such as magnetization [18] and heat capacity [19], and spectroscopic probes such as neutron scattering [20-22] and electron-spin resonance [23,24]. These strategies have been very successful in determining the energy spectra and magnetic structures, but apart from a limited number of observations of "demagnetization tunneling" there are few [25] reports of definitive measurements of relaxation times. However, the feasibility of many of the most interesting proposed applications, in particular, those involving classical or quantum information processing, is critically dependent on the intrinsic spin-lattice $\left(T_{1}\right)$ and phase-coherence $\left(T_{2}\right)$ times.

Measurements were performed using commercial Bruker Elexsys $580 \mathrm{X}$-band pulsed ESR spectrometers, employing ${ }^{4} \mathrm{He}$ flow cryostats for temperature control. The relaxation times were obtained using standard techniques [26]: $T_{2}$ from the decay of a 2-pulse Hahn-echo sequence,

$$
\pi / 2-\tau-\pi-\tau-\text { echo, }
$$

with $\tau$ varying; $T_{1}$ from the recovery of the magnetization (measured with a spin echo) after an inversion pulse,

$$
\pi-T-\pi / 2-\tau-\pi-\tau-\text { echo, }
$$

with $T$ varying and $\tau$ fixed and short.

Two factors conspire to complicate the measurement of intrinsic lifetimes in anisotropic magnetic clusters. First, in 
a crystal the magnetic cores are typically separated by rather small distances of the order of $1 \mathrm{~nm}$ to $10 \mathrm{~nm}$, and are therefore coupled by dipolar interactions. For two free electron spins, the dipolar interaction is of the order of $100 / r^{3} \mathrm{MHz} \cdot \mathrm{nm}^{3}$ [26], so this dipolar coupling is the dominant relaxation mechanism limiting $T_{2}$ in crystals of clusters. Second, clusters tend to exhibit strongly axial behavior with significant zero-field splittings; this lifts the degeneracy of the $\Delta m_{S}= \pm 1$ transitions in high spin $(S>1 / 2)$ molecules, and leads to a strong dependence of the transition energies on the orientation with respect to the external magnetic field. Thus if we are to study a particular transition in an ensemble of identical molecules, they must be orientationally ordered. The standard approach to solving the first problem, dissolving the crystal (thereby increasing the average separation of the clusters), leads to a second problem, an ensemble of randomly oriented, highly axial molecules.

We chose the two compounds studied here with these factors in mind. $\mathrm{Cr}_{7} \mathrm{Ni}$ has a ground state spin of $S=1 / 2$, so it exhibits a single ESR transition and, therefore, no zero-field splitting; the anisotropy of the $g$ factor, which is small, is the only contribution to a dependence of the transition energy on external magnetic field orientation. A dilute dissolved sample of this material is therefore amenable to ESR measurements without causing problems associated with the orientational disorder. $\mathrm{Cr}_{7} \mathrm{Mn}$ is a very closely related compound with a ground state spin of $S=$ 1; the similarity in its structure leads us to expect that it should share relaxation mechanisms with $\mathrm{Cr}_{7} \mathrm{Ni}$, but its higher spin allows us to examine the consequences of the zero-field splitting. For example, a modulation of the zero-field splitting, which might occur as a result of coupling with thermally excited mechanical deformations of the molecule, provides further spin-lattice and phasecoherence relaxation mechanisms [27].

Samples were prepared as reported elsewhere [17], and dissolved in toluene. The solution was diluted progressively until the results reported below were no longer dependent on concentration, indicating that the dipolar coupling between clusters had become negligible; this occurred for concentrations below approximately $0.2 \mathrm{mg} / \mathrm{ml}$, corresponding to a mean separation of clusters in excess of about $25 \mathrm{~nm}$.

Figure 1 shows echo-detected ESR as a function of the applied magnetic field measured at $4.5 \mathrm{~K}$ for (a) $\mathrm{Cr}_{7} \mathrm{Ni}$ and (b) $\mathrm{Cr}_{7} \mathrm{Mn}$. The spectrum for $\mathrm{Cr}_{7} \mathrm{Ni}$ shows a single narrow line, as expected for an $S=1 / 2$ species. The line shape is approximately Gaussian, with a width of about $0.01 \mathrm{~T}$, suggesting that the broadening is due to inhomogeneity of the cluster environment. In contrast, the spectrum for $\mathrm{Cr}_{7} \mathrm{Mn}$ is broad and contains structure that is characteristic of an $S=1$ species with a zero-field splitting exceeding the microwave energy. Also shown is a simulated powder spectrum [28] for an $S=1$ species with the Hamiltonian

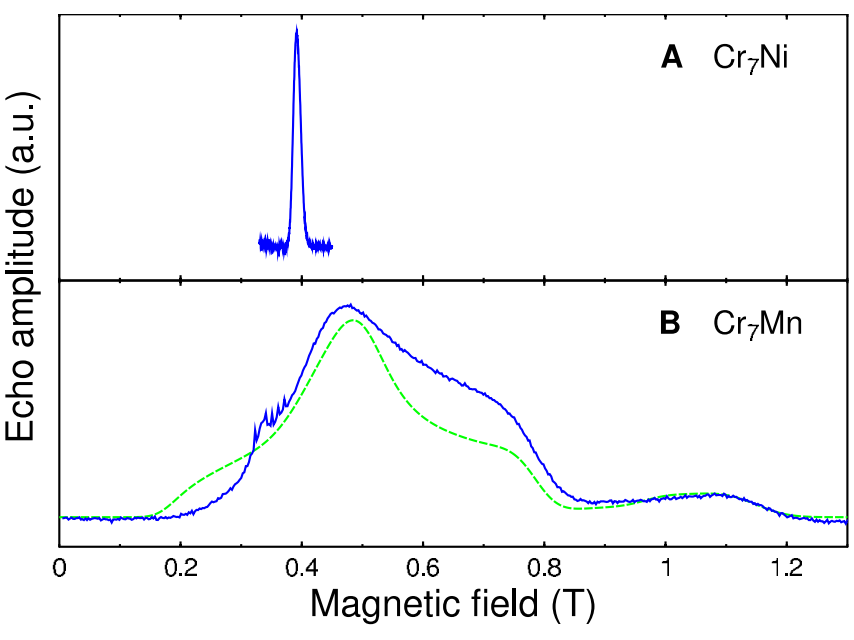

FIG. 1 (color online). $\quad X$-band echo-detected ESR as a function of magnetic field for (a) $\mathrm{Cr}_{7} \mathrm{Ni}$ and (b) $\mathrm{Cr}_{7} \mathrm{Mn}$ measured at $4.5 \mathrm{~K}$ (blue), and the simulated powder spectrum for a species with $S=1, g=2, D=21 \mathrm{GHz}, E=1.9 \mathrm{GHz}$ (green). In this experiment, the intensity of a Hahn-echo signal with short $(\tau=$ $300 \mathrm{~ns}$ ) delays is measured as a function of the applied magnetic field. Selective pulses, of $64 \mathrm{~ns}$ for a $\pi / 2$ pulse and $128 \mathrm{~ns}$ for a $\pi$ pulse, ensure that only spins within a window of about $0.3 \mathrm{mT}$ are excited. Using a broad integration window suppresses ${ }^{1} \mathrm{H}$ ESEEM effects. The echo intensity is proportional to the ESR excitation spectrum. The fine structure in the data close to $0.33 \mathrm{~T}$ is an artifact of the cavity.

$$
\mathcal{H}=g \mu \mathbf{B} \cdot \mathbf{S}+D S_{z}^{2}+E\left(S_{x}^{2}-S_{y}^{2}\right)
$$

with parameters $g=1.9, D=21 \mathrm{GHz}$, and $E=1.9 \mathrm{GHz}$, which reproduces the main features of the data [green line in Fig. 1(b)]; deviations are probably due to the fact that in the experiment, the pulses deviate from perfect $\pi$ and $\pi / 2$ rotations at different parts of the spectrum because the transition probabilities depend strongly on orientation. Both spectra show some low-amplitude fine structure close to $0.33 \mathrm{~T}$; these lines arise from impurities in the cavity and are present in the absence of the sample.

Figure 2(a) shows the intensity of Hahn echoes measured in $\mathrm{Cr}_{7} \mathrm{Ni}$ as a function of the delay time $\tau$ [defined in Eq. (1)], for long, selective pulses (blue) and short, broadband pulses (cyan). The echo generated by broadband pulses exhibits very strong modulations (electron-spin echo envelope modulation, or ESEEM) with some second harmonic content, associated with coupling of the electron spin to nearby nuclear moments [26]. The nuclear frequency extracted from the modulation, $16.6 \pm 0.1 \mathrm{MHz}$, indicates that the relevant nuclei are protons. This ESEEM can be suppressed by using longer, selective pulses, as shown by the blue line in Fig. 2(a); this echo decay is well described by a monoexponential fit (green dashed line), yielding a coherence time of $T_{2}=379 \pm 1 \mathrm{~ns}$ at $4.5 \mathrm{~K}$.

The strong coupling to protons may provide an efficient phase decoherence path, reducing $T_{2}$ [29]. A direct test of 


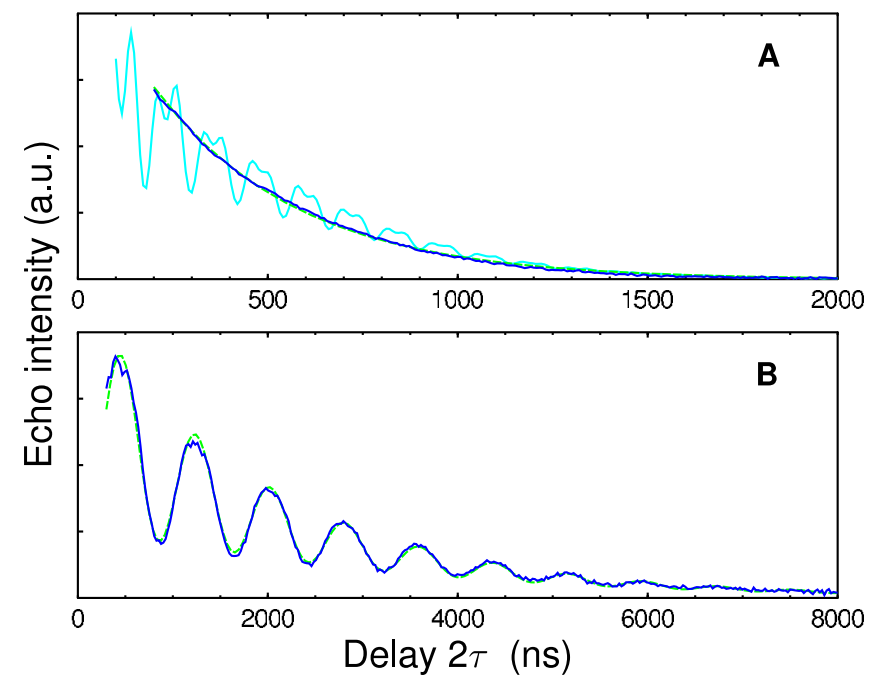

FIG. 2 (color online). (a) Decay of the Hahn-echo intensity in $\mathrm{Cr}_{7} \mathrm{Ni}$ as a function of delay time for long, selective pulses (64 ns $\pi / 2$ pulse, $128 \mathrm{~ns} \pi$ pulse, blue) and for short broadband pulses (16 ns $\pi / 2$ pulse, $32 \mathrm{~ns} \pi$ pulse, cyan). (b) Decay of the Hahnecho intensity for long, selective pulses in perdeuterated $\mathrm{Cr}_{7} \mathrm{Ni}$. Dashed green lines indicate fits to the data. The fit in (b) assumes that the ESEEM effect is dominated by a single harmonic at the ${ }^{2} \mathrm{D}$ Zeeman frequency [31]. (Note the different horizontal axis scales.)

whether this is indeed an important relaxation mechanism is to measure the same Hahn-echo decay in the perdeuterated analogue compound, as shown in Fig. 2(b). ${ }^{2} \mathrm{D}$ has a gyromagnetic ratio about 6 times smaller than ${ }^{1} \mathrm{H}$; the ESEEM frequency is correspondingly about 6 times smaller at $2.556 \pm 0.005 \mathrm{MHz}$, and $T_{2}$ is about 6 times longer, at $2210 \pm 20 \mathrm{~ns}$. This confirms that the coupling to protons dominates the spin decoherence in the hydrogenated sample. (Note that we were unable to decrease the bandwidth of the pulses sufficiently to suppress the lower frequency ESEEM in the deuterated compound.)

Figure 3 shows the temperature dependences of $T_{1}$ and $T_{2}$ for each compound. $T_{1}$ is comparable between the $\mathrm{Cr}_{7} \mathrm{Ni}$ and $\mathrm{Cr}_{7} \mathrm{Mn}$, and increases rapidly as the temperature falls. This suggests that thermal processes (such as couplings to phonons) are responsible for the longitudinal relaxation. At low temperatures, there is a variation of a factor of about two in $T_{1}$ measured at different points in the spectrum of $\mathrm{Cr}_{7} \mathrm{Mn}$. Exciting different parts of the powder spectrum corresponds to selecting subpopulations of molecules by orientation. These orientational subpopulations relax at different rates, showing that the magnetic anisotropy plays at least some role in the longitudinal relaxation, probably through spectral diffusion effects [30]. There is no such variation of $T_{2}$.

In each compound, $T_{2}$ also increases as the temperature is decreased, though less dramatically than $T_{1}$, and there are signs that it begins to saturate at temperatures below about $2 \mathrm{~K}$. There are two interesting observations: first,

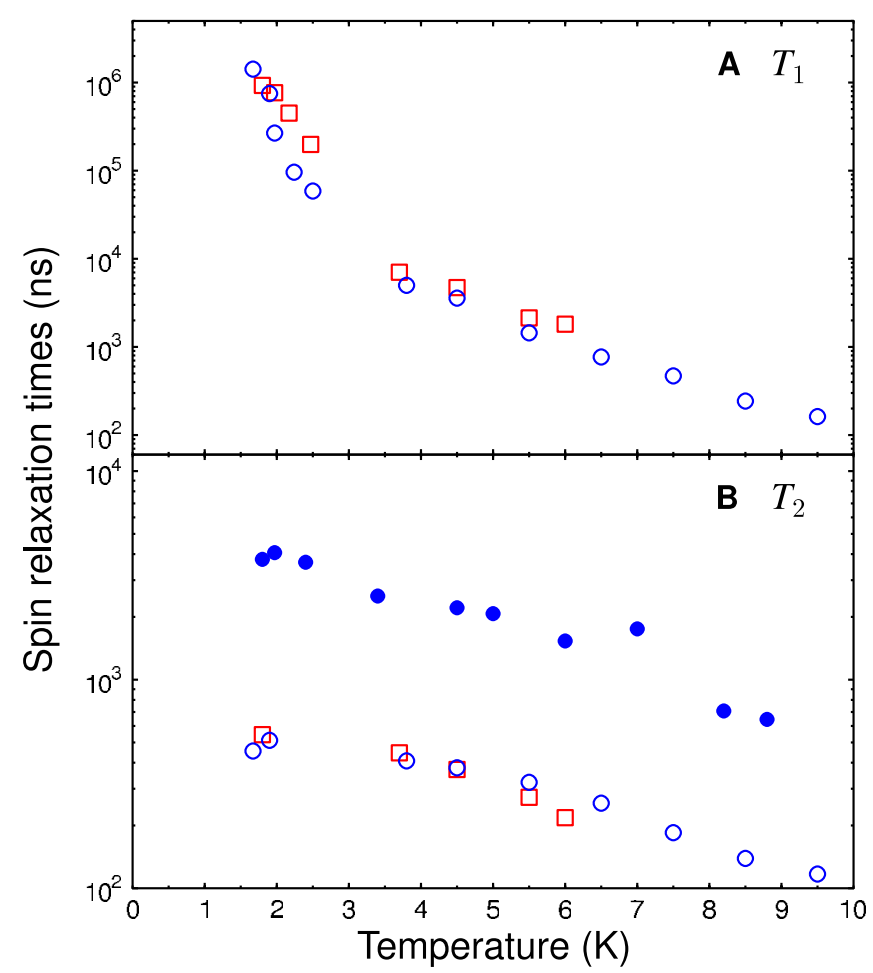

FIG. 3 (color online). (a) $T_{1}$ as a function of temperature in $\mathrm{Cr}_{7} \mathrm{Ni}$ (blue open circles) and $\mathrm{Cr}_{7} \mathrm{Mn}$ (red open squares). (b) $T_{2}$ as a function of temperature for $\mathrm{Cr}_{7} \mathrm{Ni}$ (blue open circles), $\mathrm{Cr}_{7} \mathrm{Mn}$ (red open squares), and perdeuterated $\mathrm{Cr}_{7} \mathrm{Ni}$ (blue filled circles).

there is very little difference between the hydrogenated $\mathrm{Cr}_{7} \mathrm{Ni}$ and $\mathrm{Cr}_{7} \mathrm{Mn}$, despite the strong magnetic anisotropy of the latter; second, the factor of about six between the decoherence times for the perdeuterated and hydrogenated $\mathrm{Cr}_{7} \mathrm{Ni}$ is retained over the whole temperature range over which a Hahn echo is measurable. Both observations support the hypothesis that dipolar coupling with ${ }^{1} \mathrm{H}$ or ${ }^{2} \mathrm{D}$ nuclei (which belong to organic ligands of the magnetic cluster and are well-distributed about the core [17] ) dominates the phase decoherence in these materials. Phase decoherence arising from fluctuations of the zero-field splitting in $\mathrm{Cr}_{7} \mathrm{Mn}$ is negligible in comparison over the temperature range studied here.

In conclusion, we have measured intrinsic spin-lattice $\left(T_{1}\right)$ relaxation times and, for the first time (to the best of our knowledge), the phase-coherence $\left(T_{2}\right)$ relaxation times in molecular magnets. We find that in the heterometallic clusters $\mathrm{Cr}_{7} \mathrm{Ni}$ and $\mathrm{Cr}_{7} \mathrm{Mn}, T_{1}$ is long and somewhat dependent on the magnetic anisotropy of the cluster, but that $T_{2}$ is dominated by the coupling to the nuclear moments of protons in the vicinity of the cluster. There is no evidence of coupling between the magnetic cluster and the fluorine nuclei, which had previously been identified as a potential decoherence path [9]. Futhermore, we find that the intrinsic phase-coherence time $T_{2}$ exceeds previous (worst-case) expectations by 3 orders of magnitude, reaching $0.55 \mu \mathrm{s}$ 
at $1.8 \mathrm{~K}$, and $3.8 \mu$ s for the perdeuterated analogue. With existing apparatus, the time scale for coherent manipulations of the electron spin is of the order of $10 \mathrm{~ns}$; if heteromagnetic clusters of this class were to be used as elements of a quantum information processing device, this would lead to a single-qubit figure of merit of several hundred. The identification of coupling to the ${ }^{1} \mathrm{H}$ or ${ }^{2} \mathrm{D}$ nuclei as the main decoherence path offers a strategy for synthesizing structures with even better coherence properties by reducing as far as possible the number of hydrogens and other magnetic nuclei in the vicinity of the cluster. Our results are very encouraging for the prospects of constructing and manipulating nontrivial quantum states within individual clusters $[7,8]$ and between clusters in composites [11].

We would like to thank C. Kay of University College London, and S. A. Lyon of Princeton University for the use of their spectrometers and helpful discussions. This work was supported by EPSRC Grants No. GR/S57396/01, No. GR/T27341/01, and No. EP/D048559/1. A. A. is supported by the Royal Society. J. J. L. M. is supported by St. John's College, Oxford.

[1] M. A. Nielsen and I. L. Chuang, Quantum Computation and Quantum Information (Cambridge University Press, Cambridge, England, 2000).

[2] B. Kane, Nature (London) 393, 133 (1998).

[3] D. Loss and D.P. DiVincenzo, Phys. Rev. A 57, 120 (1998).

[4] R. Vrijen, E. Yablonovitch, K. Wang, H.W. Jiang, A. Balandin, V. Roychowdhury, T. Mor, and D. DiVincenzo, Phys. Rev. A 62, 012306 (2000).

[5] W. Harneit, Phys. Rev. A 65, 032322 (2002).

[6] J. J.L. Morton, A. M. Tyryshkin, A. Ardavan, S.C. Benjamin, K. Porfyrakis, S. A. Lyon, and G. A.D. Briggs, Nature Phys. 2, 40 (2006).

[7] M. Leuenberger and D. Loss, Nature (London) 410, 789 (2001).

[8] J. Tejada, E. Chudnovsky, E. del Barco, J. Hernandez, and T. Spiller, Nanotechnology 12, 181 (2001).

[9] F. Troiani, A. Ghirri, M. Affronte, S. Carretta, P. Santini, G. Amoretti, S. Piligkos, G. Timco, and R. E. P. Winpenny, Phys. Rev. Lett. 94, 207208 (2005).

[10] S. J. Blundell and F. L. Pratt, J. Phys. Condens. Matter 16, R771 (2004).

[11] S. Hill, R.S. Edwards, N. Aliaga-Alcalde, and G. Christou, Science 302, 1015 (2003).
[12] D. Gatteschi, R. Sessoli, and J. Villain, Molecular Nanomagnets (Oxford University Press, New York, 2006).

[13] R. Sessoli, D. Gatteschi, A. Caneschi, and M. Novak, Nature (London) 365, 141 (1993).

[14] T. Lis, Acta Crystallogr. Sect. B 36, 2042 (1980).

[15] N. V. Gerbeleu, Y.T. Struchkov, G.A. Timco, A. S. Batsanov, K. M. Indrichan, and G. A. Popovich, Dokl. Akad. Nauk SSSR 313, 1459 (1990).

[16] J. van Slageren, R. Sessoli, D. Gatteschi, A. A. Smith, M. Helliwell, R. E. P. Winpenny, A. Cornia, A.-L. Barra, E. R. Aloysius G. M. Jansen, and G. A. Timco, Chem. Eur. J. 8, 277 (2002).

[17] F. Larsen, E. McInnes, H.E. Mkami, J. Overgaard, S. Piligkos, G. Rajaraman, E. Rentschler, A. Smith, G. Smith, and V. Boote et al., Angew. Chem, Int. Ed. 42, 101 (2003).

[18] A. Cornia, D. Gatteschi, and R. Sessoli, Coord. Chem. Rev. 219-221, 573 (2001).

[19] F. Fominaya, J. Villain, P. Gandit, J. Chaussy, and A. Caneschi, Phys. Rev. Lett. 79, 1126 (1997).

[20] I. Mirebeau, M. Hennion, H. Casalta, H. Andres, H. U. Güdel, A. V. Irodova, and A. Caneschi, Phys. Rev. Lett. 83, 628 (1999).

[21] M. Hennion, L. Pardi, I. Mirebeau, E. Suard, R. Sessoli, and A. Caneschi, Phys. Rev. B 56, 8819 (1997).

[22] R. Caciuffo, T. Guidi, G. Amoretti, S. Carretta, E. Liviotti, P. Santini, C. Mondelli, G. Timco, C. A. Muryn, and R. E. P. Winpenny, Phys. Rev. B 71, 174407 (2005).

[23] D. Gatteschi, A.L. Barra, A. Caneschi, A. Cornia, R. Sessoli, and L. Sorace, Coord. Chem. Rev. 250, 1514 (2006).

[24] E. McInnes, Structure and Bonding (Berlin) 122, 69 (2006) gives a comprehensive account of spectroscopy (ESR, NMR, and INS) of single molecule magnets.

[25] W. Wernsdorfer, D. Mailly, G. A. Timco, and R.E.P. Winpenny, Phys. Rev. B 72, 060409 (2005).

[26] A. Schweiger and G. Jeschke, Principles of Pulse Electron Paramagnetic Resonance (Oxford University Press, New York, 2001).

[27] A. Carrington and G. G. R. Luckhurst, Mol. Phys. 8, 125 (1964).

[28] S. Stoll and A. Schweiger, J. Magn. Reson. 178, 42 (2006).

[29] N. V. Prokof'ev and P. C. E. Stamp, Rep. Prog. Phys. 63, 669 (2000).

[30] S. S. Eaton and G. R. Eaton, in Relaxation Times of Organic Radicals and Transition Metal Ions, edited by G. R. Eaton, S.S. Eaton, and L.J. Berliner, Biological Magnetic Resonance Vol. 19 (Springer-Verlag, Berlin, 2000), pp. 29-154.

[31] S. A. Dikanov and Yu. D. Tsvetkov, Electron Spin Echo Envelope Modulation (ESEEM) Spectroscopy (CRC Press, Boca Raton, 1992). 